\title{
Experimental and computational investigation of an 'open' transonic cavity flow
}

\author{
K Atvars $^{1}$, K Knowles ${ }^{1 *}$, S A Ritchie ${ }^{1}$, and N J Lawson ${ }^{2}$ \\ ${ }^{1}$ Aeromechanical Systems Group, Cranfield University, Shrivenham, UK \\ ${ }^{2}$ National Flying Laboratory Centre, Cranfield University, Bedfordshire, UK
}

The manuscript was received on 13 August 2008 and was accepted after revision for publication on 17 March 2009.

DOI: 10.1243/09544100JAERO445

\begin{abstract}
This paper presents an investigation of a transonic flow $\left(M_{\infty}=0.85\right)$ over a rectangular cavity having a length-to-depth ratio of 5 . Velocities were measured inside the cavity on the central plane and two off-centre planes using a two-component particle image velocimetry system. These measurements were supported by surface flow visualization, and mean and time-varying surface pressure measurements. The flow was also simulated using an unsteady Reynolds-averaged Navier-Stokes code, with a realizable $\kappa-\varepsilon$ turbulence model. It is shown that this CFD model does not capture all the characteristics of the flowfield correctly. However, by using this integrated experimental and computational approach we have been able to identify three-dimensional flowfield structures within the cavity. The influence of the thickness of the approaching boundary layer is discussed.
\end{abstract}

Keywords: transonic cavity, flow visualization, particle image velocimetry (PIV), unsteady Reynolds-averaged Navier-Stokes (URANS), CFD

\section{INTRODUCTION}

The phenomenon of flow within a rectangular cavity immersed in transonic flow has become the focus of much research interest recently due to the importance of stealth (and aerodynamic efficiency) in future manned aircraft, such as the F-35 Lightning II, and various unmanned combat air vehicle projects. These aircraft are designed such that the internal carriage of weapons is vital in maintaining a low radar crosssection, which in turn increases the vehicle's survivability. However, when the weapons bay doors are opened for weapons release, flow over the exposed cavity can cause a number of undesirable effects. These include self-sustaining acoustic oscillations and high-intensity tones that can lead to structural fatigue [1] (occurring, primarily, in cavities with a low ratio of streamwise length, $L$, to cavity depth, $H$ ) and adverse longitudinal pressure distributions leading to nose-in pitching moments on stores released from the cavity (primarily for high $L / H$ ) [2].

\footnotetext{
* Corresponding author: Cranfield University, Defence Academy of the UK, Shrivenham, Swindon, Wiltshire SN6 8LA, UK. email:k.knowles@cranfield.ac.uk
}

As suggested above, rectangular cavity flows can be defined as one of two main types, primarily dependent on the length-to-depth ratio $(L / H)$ of cavity [3] (see Fig. 1). 'Open' cavity flows (Fig. 1(a)) occur in cavities with $L / H<7-9$ (typically) and are characterized by strong pressure oscillations that lead to noise radiation (often in excess of $170 \mathrm{~dB}$ ), structural vibration, and high levels of heat transfer at the trailing edge. 'Closed' cavity flows (Fig. 1(b)) occur in cavities with $L / H>10-14$ (typically) and are regarded as quasi-steady flows. The pressure distribution along the floor of a 'closed' cavity shows a large longitudinal pressure gradient (Fig. 1(c)) that causes a large increase in pressure drag and can lead to store separation difficulties. Cavity geometries in the approximate range $8<L / H<11$ are described as 'transitional' and here the cavity flows exhibit a combination of 'open' and 'closed' flow features (Fig. 1(c)). Note that Plentovich et al. [3] found that the precise boundaries between open, transitional, and closed flows (defined by the centre-line pressure distribution) depend on freestream Mach number and cavity width-to-depth ratio $(W / H)$. Nevertheless, open flow always occurred for $L / H<7$.

For 'open' cavity flows, the pressure oscillations that are observed were initially investigated by Rossiter [4]. 


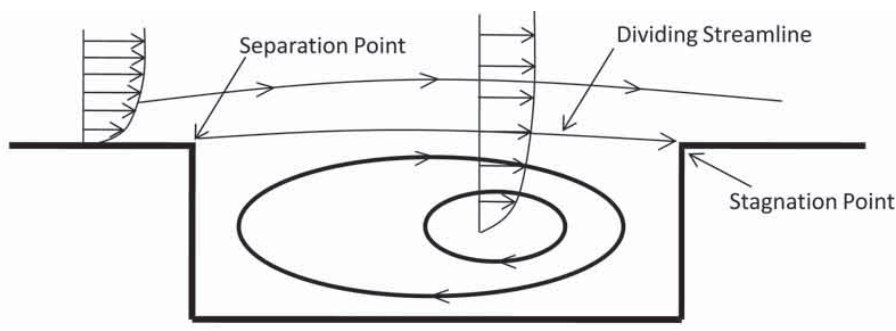

(a)

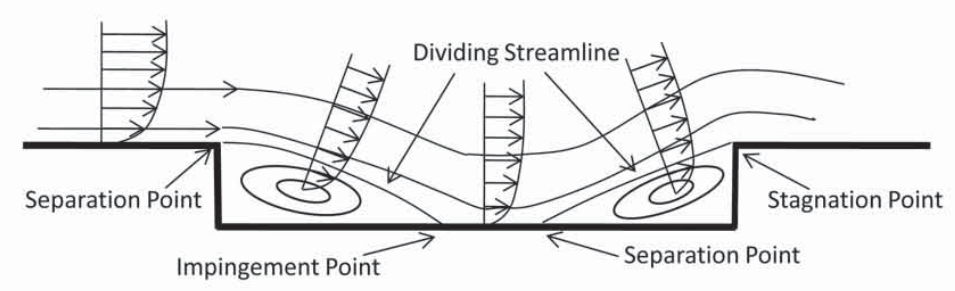

(b)

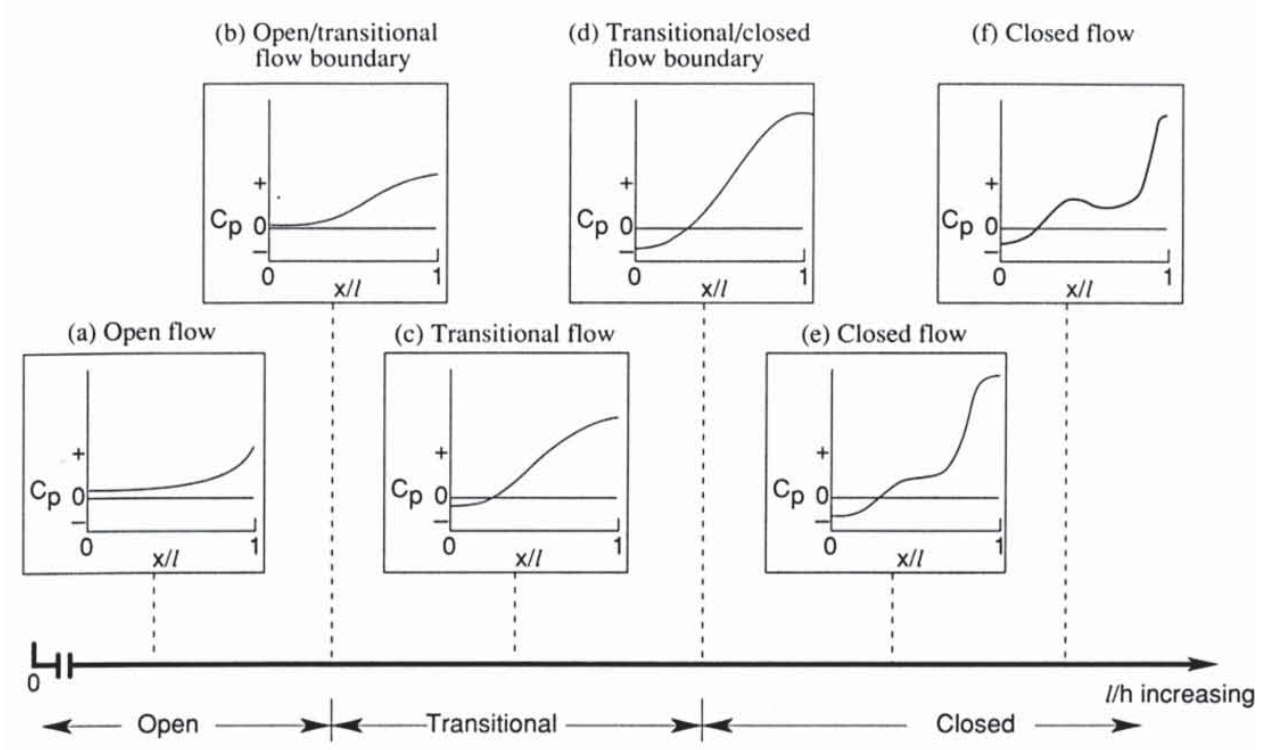

(c)

Fig. 1 Cavity flow types: (a) schematic of subsonic, open cavity flow; (b) schematic of subsonic, closed cavity flow; and (c) classification for subsonic flows, based on streamwise pressure gradient, after Stallings et al. [2]

Rossiter proposed a feedback loop, whereby acoustic waves would form at the downstream wall from vortices shed initially from the upstream cavity wall, with synchronization occurring between points in the loop. This resulted in the semi-empirical 'Rossiter equation', which can be used to predict the frequency for a given mode of oscillation in a given cavity geometry

$$
f=\frac{U_{\infty}}{L} \frac{(m-\alpha)}{\left(M_{\infty}+(1 / K)\right)}
$$

This was subsequently modified [5] to account for the higher speed of sound within the cavity, which is approximately equal to the freestream stagnation speed of sound. This 'modified Rossiter equation' is used here to predict the frequencies of the various oscillation modes in an open cavity flow

$$
f=\frac{U_{\infty}}{L} \frac{(m-\alpha)}{M_{\infty}\left[1+((\gamma-1) / 2) M_{\infty}^{2}\right]^{-1 / 2}+(1 / K)}
$$

where $\alpha$ is an empirical constant, related to the phase lag between instabilities in the shear layer and upstream-travelling pressure wave, which is dependent on the cavity length-to-depth ratio and is given by $\alpha=0.062(L / H)$.

The constant $K$ is the empirical ratio of shear layer and freestream velocities; $K=0.57$ is appropriate for thin initial boundary layers but decreases with increasing boundary layer thickness. 
Many previous studies of cavity flows have concentrated on the time-averaged and unsteady measurement of the flow using static pressure taps on the surfaces and qualitative visualization techniques such as schlieren imagery and oil flow visualization [6-8]. The results of these studies have typically been compared with numerical models with mixed success $[\mathbf{9}, \mathbf{1 0}]$. There is currently very little data available on the off-surface flowfield within different cavity geometries under transonic conditions. Despite recent improvements in optical measurement techniques such as particle image velocimetry (PIV) $[11,12]$ and laser Doppler anemometry (LDA) [13], there appears to be little, if any, quantitative flow measurement of the structure of a transonic cavity flow that is not confined to the centre-line of the cavity [14]. In this investigation, we present results of applying PIV measurement techniques to a transonic cavity flow and incorporate results from surface pressure measurements, surface flow visualization, and numerical simulation to produce an overall image of the flow structure in a transonic cavity flow.

\section{EXPERIMENTATION}

All tests were conducted using the Shrivenham transonic wind tunnel (TWT), which has a working section of $206 \mathrm{~mm}$ (high) by $229 \mathrm{~mm}$ (wide). This is a closed circuit, ejector-driven tunnel supplied with air from two Howden screw-type compressors. The compressors supply air at up to $7 \mathrm{bar}(\mathrm{g})$, which is dried and stored in a $34 \mathrm{~m}^{3}$ reservoir. The stored air is sufficient to run the tunnel at Mach 0.85 (the test condition for the present measurements) for about $15 \mathrm{~s}$.

The geometry of the cavity is indicated in Fig. 2, together with the co-ordinate system used here and its origin. To enable optical access for PIV measurements, an all-glass cavity was mounted from the underside of a flat plate that had a sharp leading edge and was raised some $16 \mathrm{~mm}$ away from the tunnel side wall (Fig. 3). Data could not be acquired for the first $2 \mathrm{~mm}$ of the cavity depth due to the presence of the splitter plate. Similarly, the wind tunnel design does not allow the freestream flow to be measured using PIV, because

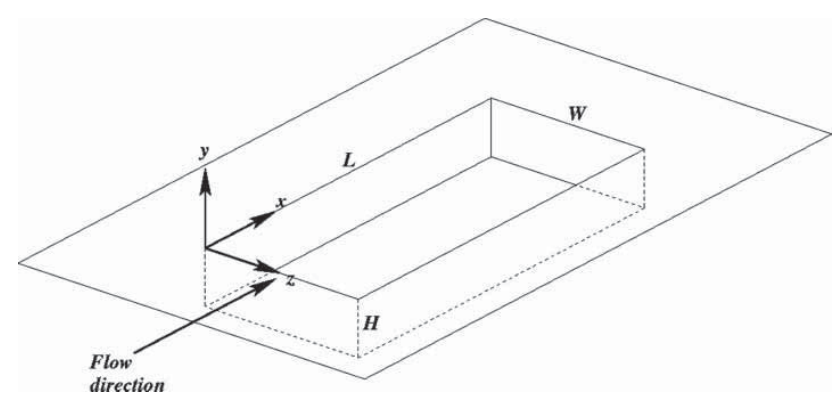

Fig. 2 Cavity geometry and axis systems

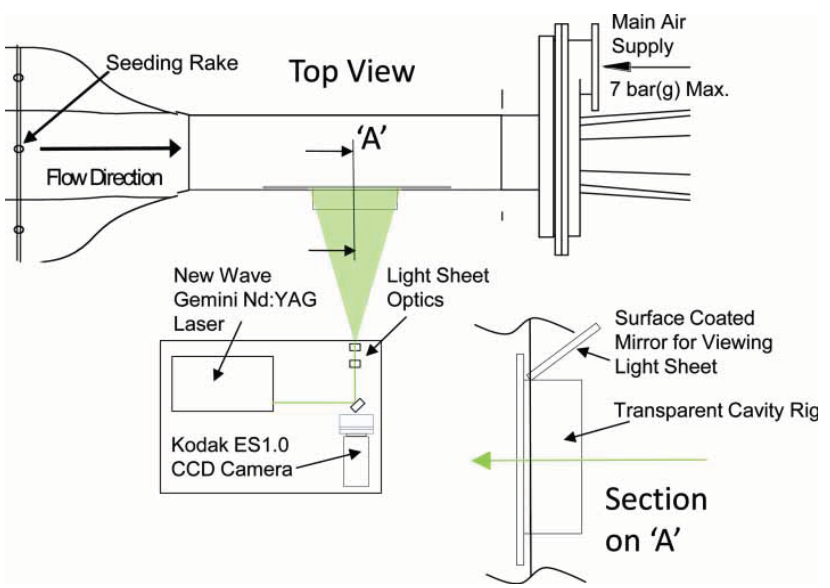

Fig. 3 PIV experimental set-up

of a lack of optical access but glass panels were fitted in the flat plate upstream and downstream of the cavity to enable LDA measurements in the freestream. For cavity floor pressure measurements and oil flow visualization, an aluminium cavity was used. In each case, the cavity had dimensions of $L=160 \mathrm{~mm}, W=$ $80 \mathrm{~mm}(L / W=2)$. For the results presented here, the cavity had a depth of $H=32 \mathrm{~mm}$, to give $L / H=5$ (other $L / H$ values were also tested but are not discussed here). This resulted in a blockage ratio of 5 per cent, which is well within acceptable limits for high-speed tunnel testing [15].

At $M_{\infty}=0.85$, a freestream turbulence intensity of 5-6 per cent was measured between 20 and $150 \mathrm{~mm}$ above the plane of the cavity $(y / H=0.63-4.7)$. A pitotstatic probe, located $30 \mathrm{~mm}$ upstream of the cavity leading edge $(x / H=-0.94)$, was used to measure the flow approaching the cavity. This revealed a turbulent boundary layer with thickness $\delta_{0.99}=18.7 \mathrm{~mm}$ $(0.584 H)$, which can be expected to grow a further $0.6 \mathrm{~mm}$ by the leading edge of the cavity. This is much thicker than the natural TWT wall boundary layer at this point $(6 \mathrm{~mm})$ and appears to be the result of a separation at the plate leading edge, followed by a turbulent reattachment.

A custom-built seeding system injected water particles of 5-10 $\mu \mathrm{m}$ diameter into the contraction section to seed the flow (Fig. 3). This size resulted in a seeding response error of 2.3 per cent of the measured velocity, using the error calculation method described by Dring [16]. The PIV acquisition system consisted of a Dantec FlowMap 500 processor, a Kodak ES1.0 CCD camera, and a New Wave Gemini Nd:YAG pulsed laser. The light sheet was projected into the cavity through the clear floor. The seeded light sheet was viewed perpendicularly via a surface-coated mirror angled at $45^{\circ}$ to the cavity right side wall (see Fig. 3). PIV data were taken for the same three $x-y$ planes across the cavity on which pressure data were acquired (see below). 
The Kodak ES1.0 camera frame rate and laser repetition rate allowed data to be recorded at up to $15 \mathrm{~Hz}$. This frame rate was too low to capture the unsteady phenomena in the cavity; hence, time-averaged flow data derived from the instantaneous data are presented from the PIV. (At least a $2 \mathrm{kHz}$ system would be needed before time-resolved data could be acquired successfully at this scale.) Time averaging was performed in correlation space, which also improved the resolution in regions where fewer particles had been entrained in the flow, such as in the cavity upstream recirculation region. A set of 700 image pairs were acquired per run at a $15 \mathrm{~Hz}$ sampling rate and processed into instantaneous vector maps using a window-deformation FFT code; these were then time averaged. To process the PIV images, DaVis software by La Vision was used. The software employs an iterative image-deformation algorithm, similar to those reviewed by Scarano [17], which more effectively extracts vectors from complex rotating flows with high velocity gradients (as seen in open cavity flows). The window-deformation technique deforms the interrogation region in the second frame according to the velocity gradient present within that region. This leads to identical displacements for all the seeding particles within a region giving an improved signalto-noise ratio and thus higher accuracy. To process the images, four passes were used with two passes at $32 \times 32$ pixels and two passes at $16 \times 16$ pixels. All regions were 75 per cent overlapped in $x$ and $y$. To aid clarity, the vector maps presented here were post-processed by sub-sampling by a factor of 3 . Based on the seeding response, the geometry error, and the processing error, estimated accuracy in this case is better than 3.4 per cent of full-scale measurement. The accuracy of the PIV measurements could have been increased by using seeding particles with sub-micron diameters; however, this would then require greater laser power to scatter an equivalent amount of light, which was not possible for the present tests.

Surface flow visualizations were performed using a paraffin-based solution containing solar yellow fluorescent paint particles. By subjecting the particles to ultraviolet light, the surface flow patterns were clearly visible and could be photographed using digital still photography. Cavity floor pressures were measured using three rows of nine pressure tappings each, at $z / W=0.5,0.667$, and 0.833 (referred to as the $C L, O C 1$, and $O C 2$ planes, respectively). These tappings were connected via $46 \mathrm{~cm}$ lengths of tubing of a known frequency response to a Scanivalve ZOC block electronically-scanned pressure transducer containing 32 piezo-resistive pressure sensors. Measurements were taken at a sample rate of $10 \mathrm{kHz}$, and filtered at $5 \mathrm{kHz}$ with a resolution of approximately $5 \mathrm{~Hz} ; 65536$ samples were taken at each of four TWT runs and averaged.

\section{COMPUTATION}

Numerical simulation data were obtained using timeaveraged unsteady, Reynolds-averaged Navier-Stokes (URANS) CFD predictions. The URANS approach

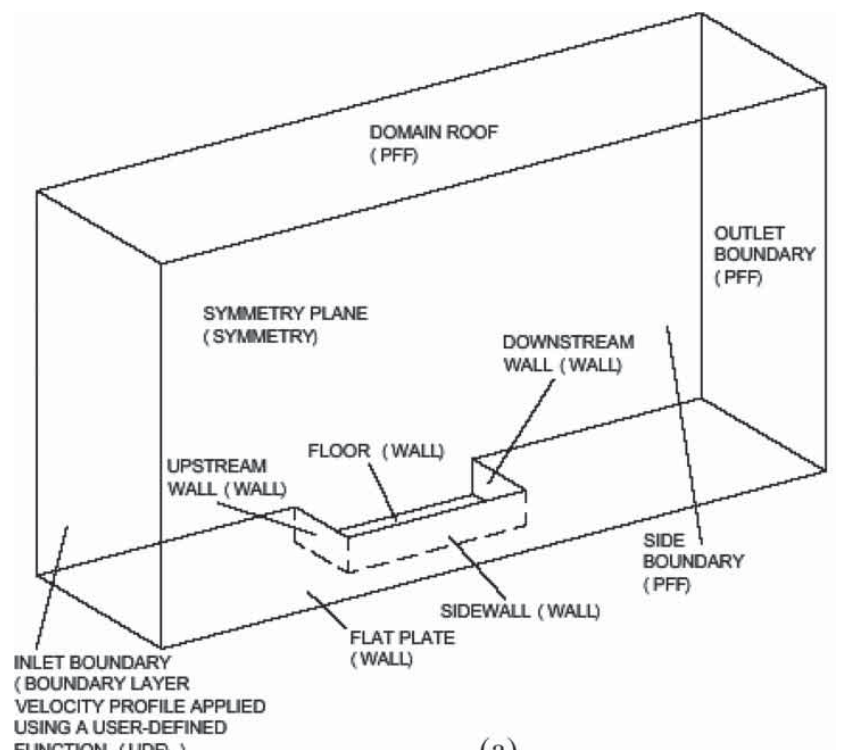

FUNCTION (UDA) )

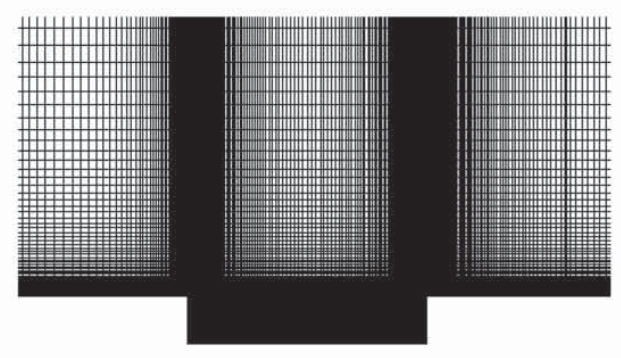

(b)

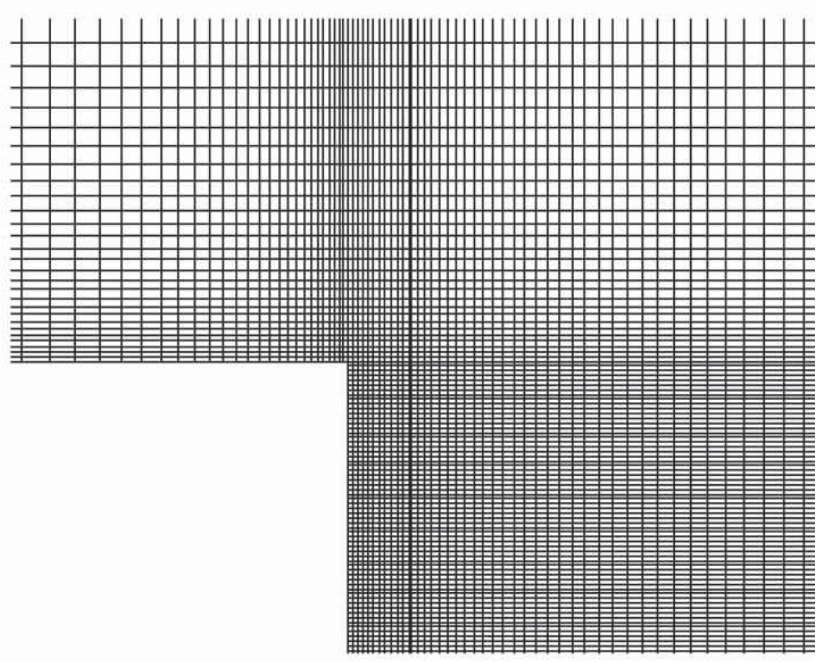

(c)

Fig. 4 CFD domain and grid: (a) domain construction and boundary type; (b) overview of grid in and around cavity; and (c) detail of grid around upstream cavity lip 
used here employed the Fluent commercial code and adopted the realizable $\kappa-\varepsilon$ turbulence model. This turbulence model was chosen as it includes modifications over the standard $\kappa-\varepsilon$ model, which makes it more suitable for use in flows containing regions of high shear and swirl [18].

Previous work has shown that open-type cavity flows are largely symmetrical about the centreline plane, when time averaged [19]. For the present work, this was confirmed by comparing full and half-domain calculations in terms of mean flow structure and mode shapes; although not identical, both agreed equally well with experiments. For most of the results presented here, therefore, only half of the domain was calculated, with symmetry imposed on the centreline plane. This greatly reducing the computational requirements, but at the expense of suppressing any lateral oscillation modes. A mesh refinement study led to the adoption of a mesh containing 952000 quadrilateral cells (for the half domain, twice this for the full domain), which was found to be sufficient to resolve accurately the Rossiter modes (see reference [20]). This mesh had an increasing number of cells closer to the edges of the cavity (Fig. 4). Approximately 20 cells were used to resolve the boundary layer profile upstream of the cavity, with a minimum cell height chosen to give $y^{+}$values of the order of unity. This boundary layer profile was specified to match the experimentally measured profile. Calculations were also performed with a much thinner, naturally growing (but tripped) turbulent boundary layer approaching the cavity [20]. The upstream and downstream domain boundaries (inlet and outlet) were located $2 L$ from the cavity leading edge and trailing edge, respectively. The distance from the surface plane to the upper domain boundary was also $2 L$, which was found to reproduce freestream flow conditions at that boundary.

The time step was defined by the sampling rate required to resolve the second Rossiter mode with 50 data points [21]. At this time step of $\Delta t=1.76 \times 10^{-5} \mathrm{~s}$, the results had also been found to have converged, based on the measurements of the first Rossiter mode. The simulation was initially run to achieve a steady solution, as determined by the static pressure at the mid-height of the downstream wall of the cavity varying by $<1$ per cent. Then the unsteady solution was attained over a further 20000 time steps, which also ensured transients from start-up had been purged.

\section{RESULTS AND DISCUSSION}

\subsection{Time-averaged pressure data}

The experimental and numerical time-averaged pressure coefficient $\left(C_{\mathrm{p}}\right)$ data are shown for the three planes within the cavity in Fig. 5. Although the general levels of $C_{\mathrm{p}}$ are similar in the two data sets, it is clear

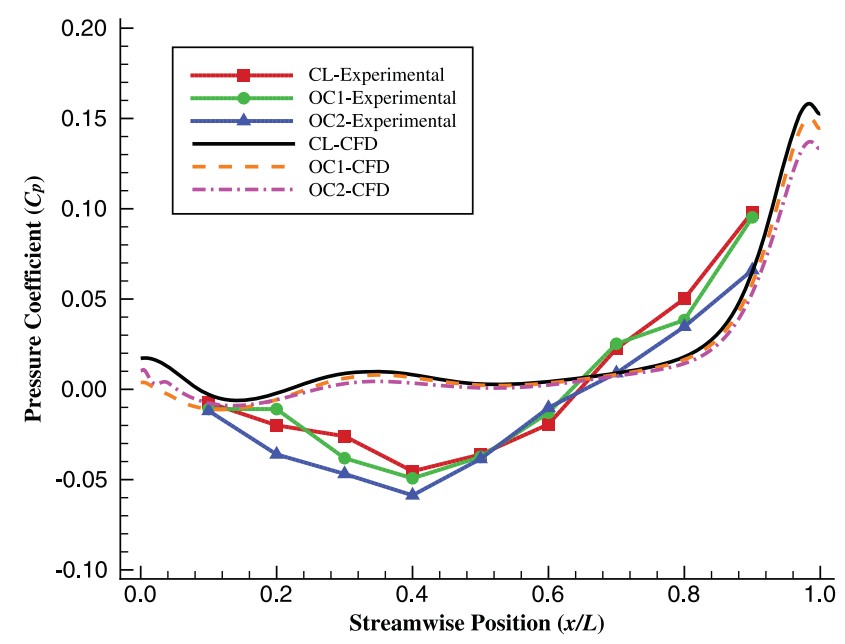

Fig. 5 Comparison of mean pressure coefficient profiles: experiment versus 3D CFD, half-domain simulation

that the form of the pressure distributions is different; this will be discussed below.

Both the experiments and the CFD show only small differences in pressure distribution between the three planes $(C L, O C 1$, and $O C 2)$. In general for both cases the further from the centreline the lower the pressure, suggesting slightly higher velocities nearer the sidewalls. This small effect is, however, less apparent in the CFD than the experiments.

The CFD results show fairly uniform pressure $\left(C_{\mathrm{P}}\right.$ close to zero) for approximately 80 per cent of the cavity length, before a sharp increase near the downstream wall. This form is typical of an open flow, as suggested by Stallings and co-workers $[2,3]$ and shown in Fig. 1. By contrast, the experimental $C_{\mathrm{p}}$ along the first 60 per cent of the cavity floor is slightly negative, reaching a minimum at approximately $x / L=0.4$. $C_{\mathrm{p}}$ continues rising over the downstream 40 per cent of the cavity length, reaching a maximum recorded value at $x / L=0.9$. These experimental pressure distributions within the cavity are typical of flow on the boundary between open-type and transitional-opentype flows (see Fig. 1). It is suggested here that this is probably due to the thick boundary layer in the present experiments. Although this effect could not be reproduced fully by the CFD, it was seen in all our experimental studies on other cavity geometries: in each case the pressure distribution was typical of a higher length-to-depth ratio than that being tested. Despite this difference (between CFD and experiments), the internal structure of the cavity flow is largely unaffected by this apparent transition [22]

\subsection{Unsteady pressure data}

Figure 6 shows the unsteady pressure spectra from the pressure tapping at $x / L=0.9$ on the $C L$ plane for 


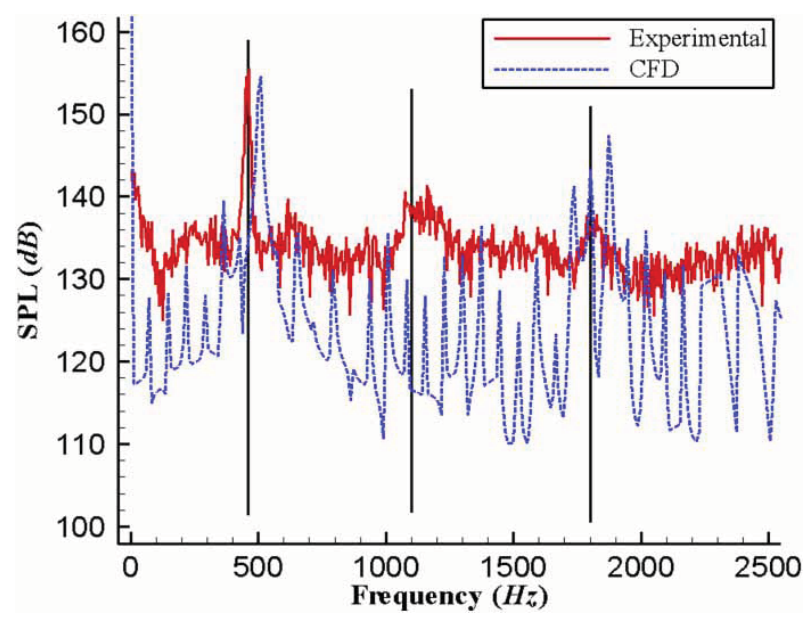

Fig. 6 Comparison of unsteady pressure spectra on centreline at $x / L=0.9$ : experiment versus $3 \mathrm{D} C F D$, half-domain simulation. Experimental spectrum has been adjusted for frequency response of apparatus. The vertical lines indicate the first three Rossiter modes according to equation (2)

the experimental and numerical cases. Both spectra show the presence of high intensity peaks within the signal, the frequencies of which are compared with the theoretical Rossiter frequencies calculated using equation (2). The agreement between the theoretical and experimental results is excellent for the first and third Rossiter modes, with the experimental data showing less than 0.5 per cent variation from the theoretical data in both cases. There is similarly close agreement for both of the off-centre planes (there is only a slight change of experimental frequency for the second and third modes). The second mode shows a broader peak in the experimental data but this is also at a similar frequency to the prediction of the modified Rossiter equation (equation (2)).

The numerical simulation shows slightly higher frequencies than the experiments for the first and third modes, but both are within about 10 per cent of the theoretical values (which do not vary with spanwise position). There is no change in first mode frequency and only a small change $(4 \mathrm{~Hz})$ in the third computed mode between the three planes. There are no second mode peaks visible in the numerical simulation spectra above the background noise level for any of the three planes. The cavity is shown to be oscillating with a first mode dominance in both the experimental and numerical cases, which suggests that the simulation has successfully predicted the oscillation feedback mechanism within the cavity. The numerical first mode peak sound pressure level (SPL) of $155 \mathrm{~dB}$ compares with $161 \mathrm{~dB}$ seen experimentally. The highest-frequency oscillation mode in both the experimental and numerical data is the third mode, after which the background noise level swamps any frequency peaks.
The issue of simulating the flow with a plane of symmetry can also be addressed indirectly by looking at unsteady pressure spectra. It is known that asymmetric flow inside a cavity correlates with a second Rossiter mode dominance in the pressure spectrum [23]. The second mode appears to be much reduced in the experimental case and appears to be completely absent in any discernable form from the CFD results. This absence of second-mode dominance in both CFD and experimental results suggests that the

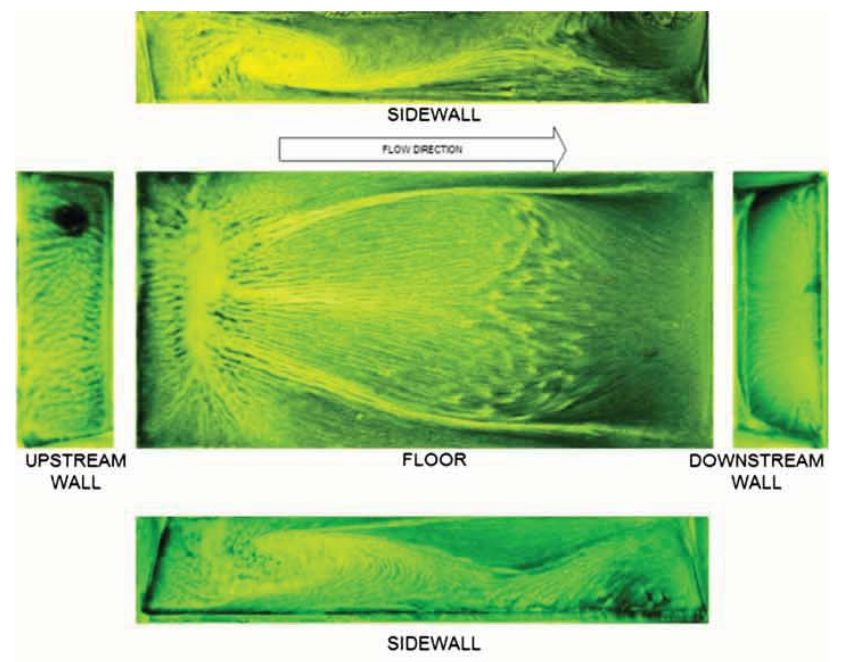

(a)

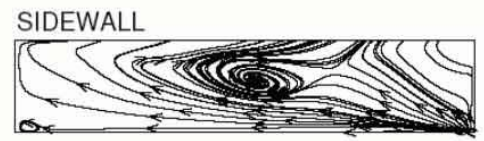

I/h=5 'Clean' Cavity Geometry - 3D Modelling Approach 1 Surface Streamlines
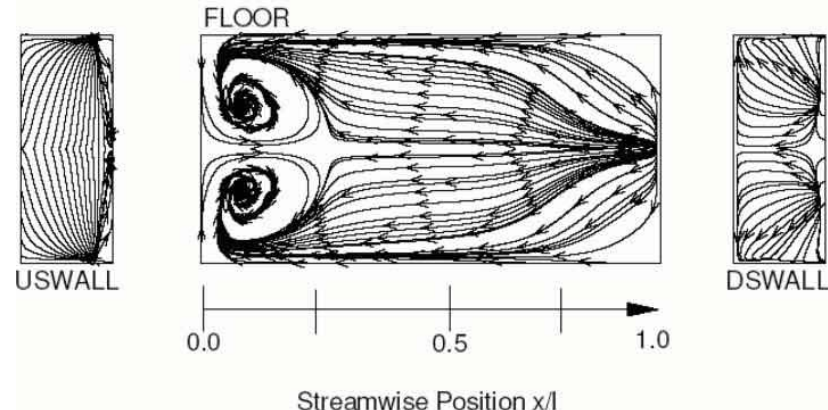

(b)

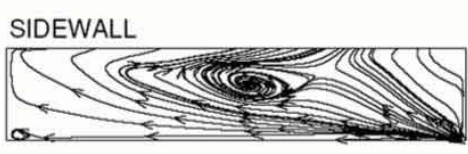

(c)

Fig. 7 Surface flow patterns: (a) experimental flow visualization; (b) surface streamlines from 3D CFD, half-domain simulation, 'thin' boundary layer; and (c) sidewall streamlines from 3D CFD, full-domain simulation, 'thin' boundary layer 
symmetry condition on the CFD domain is not affecting the predicted flow structures [23]. A full-domain three-dimensional (3D) simulation (discussed further below) has also qualitatively shown good agreement with the symmetry condition.

\subsection{Surface flow visualization}

The pressure coefficient profiles and unsteady spectra have been used to identify the type of flow and oscillation modes occurring within the cavity; a more detailed description of the flow behaviour and structure, however, is not possible from these data alone. Description of the flow behaviour can be further developed with reference to the surface flow visualization study.

The experimental and computational surface flow patterns are presented in Fig. 7. Note that the experimental images of the side and end walls (Fig. 7(a)) include some perspective error; so, in each case, the diagonal line along the edge of the image is a corner of the cavity. In both the experimental and numerical cases, the streamlines on the floor of the cavity show that the flow is travelling against the freestream direction over most of the cavity length and width, which is consistent with a large single recirculation region in the cavity rotating clockwise for a freestream flow travelling left to right.

The CFD results in Fig. 7(b) show a similar pattern on the cavity floor to the experiments, but the sidewall reveals that the main recirculation region is further downstream than that in the experiments. These CFD results have been produced with a thin, 'natural' boundary layer approaching the cavity: the imposition of the thick, experimental boundary layer produced an even more downstream position for the main recirculation. The full-domain calculations, which were only conducted with the thin boundary layer, give surface flow patterns (Fig. 7(c)) that are very similar to those seen in Fig. 7(b).

In both the experiments and the CFD, the cavity floor streamlines show two contra-rotating flow structures on either side of the centreline plane with flow rotation towards the centreline of the cavity, much like a focus sink. The structures are described in ESDU Item 02008 [24] as 'tornado-like' vortices, which spiral up towards the mouth plane (i.e. the open plane) of the cavity. Their subsequent trajectory is not speculated on in reference [24] but will be discussed further in the next section. These structures are formed when the flow travelling upstream along the floor of the cavity reaches the natural flow boundary formed by the upstream wall. The proximity
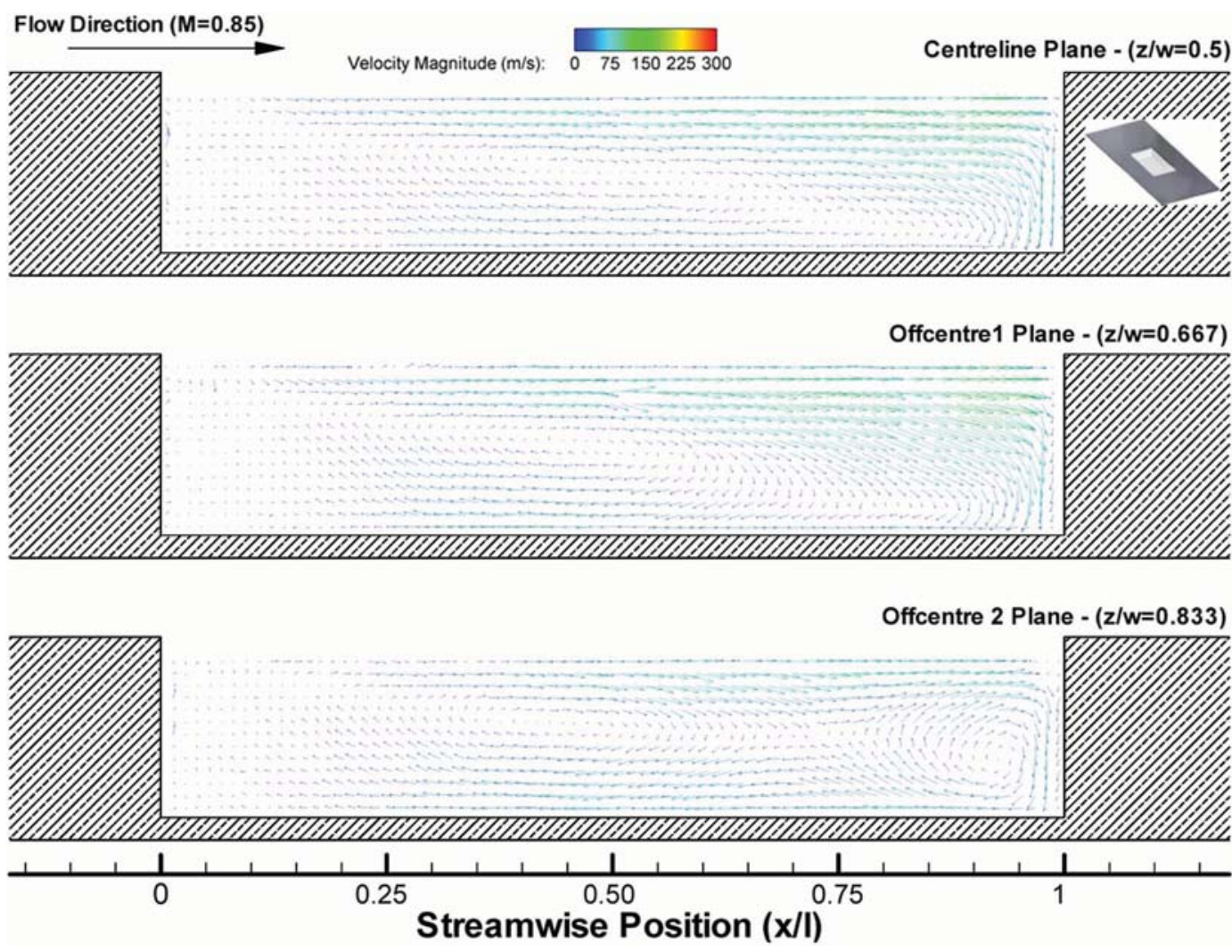

Fig. 8 PIV results: velocity vectors, coloured by magnitude, for planes CL (top) $O C 1$ (middle) and OC2 (bottom). The icon on the top right indicates that these results are for an empty cavity 
of the cavity sidewall forces the flow to divert in the spanwise direction towards the centreline of the cavity. When the flow reaches the CL plane, it meets the flow from the other side of the centreline and is forced to turn to flow downstream but is prevented from doing so by the flow travelling upstream along the cavity floor. The flow is forced to turn out towards the sidewall of the cavity, which forms the vertical 'tornado-like' structures seen on the cavity floor. Note that similar structures are still seen in 'closed'-type cavity flows (for higher length-to-depth ratios than used here) but their sense of rotation is reversed [24].

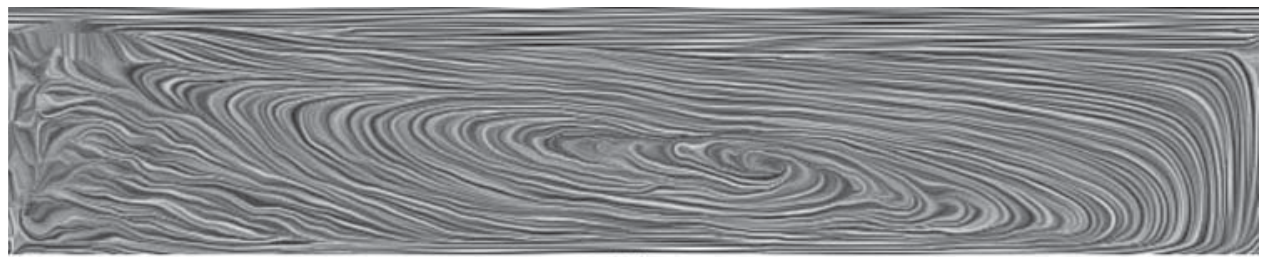

(a)

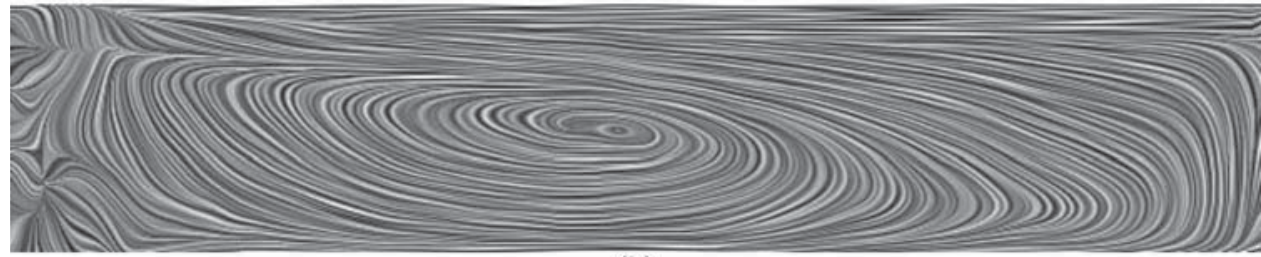

(b)

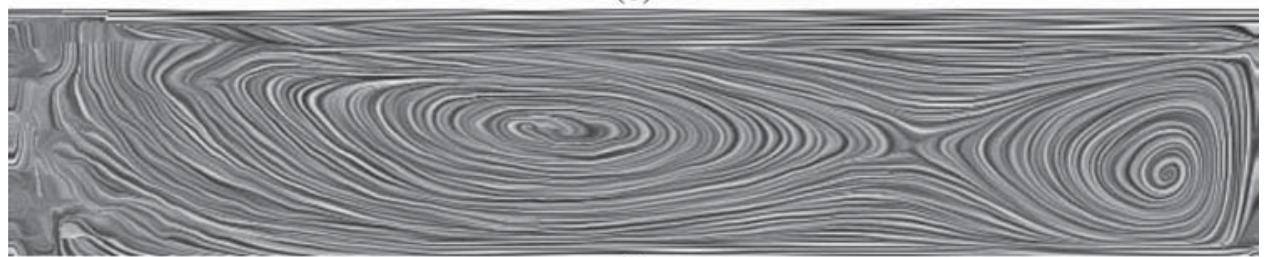

(c)

Fig. 9 LIC images of the PIV-derived velocity vector fields: (a) $C L$ plane; (b) $O C 1$ plane; and (c) OC2 plane

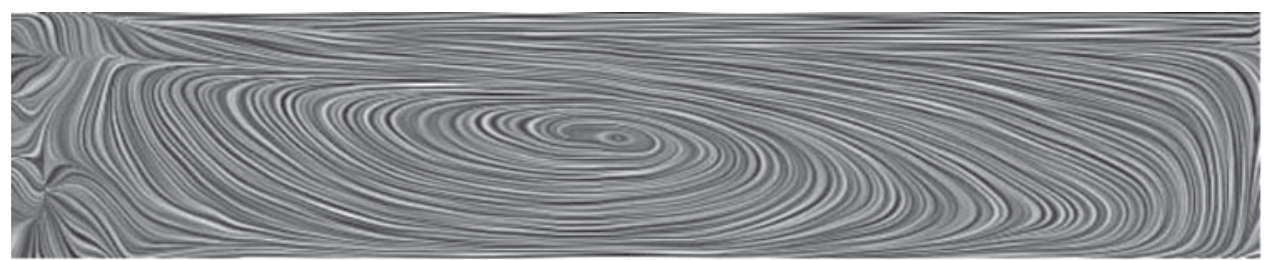

(a)

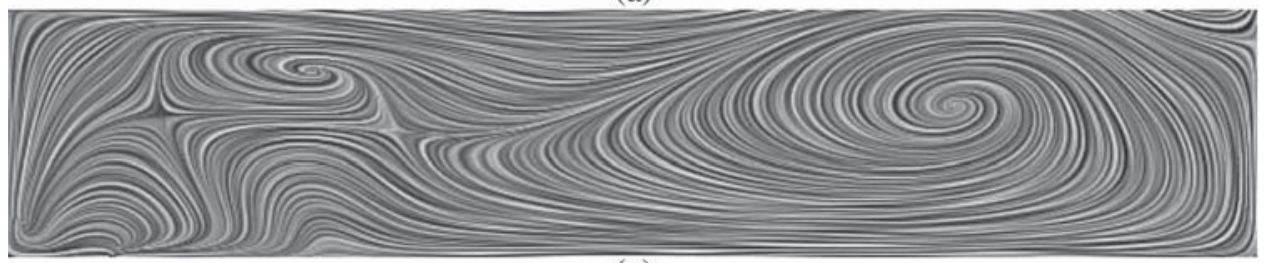

(a)

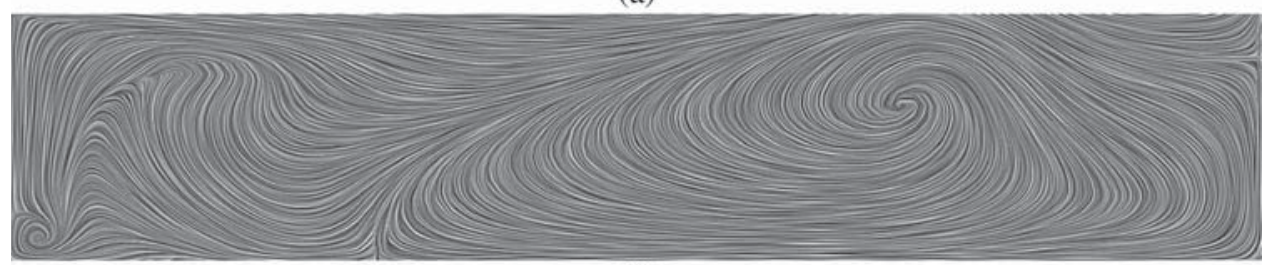

(c)

Fig. 10 LIC images of $O C 1$ plane: (a) PIV results; (b) CFD results with a half-domain simulation; and (c) CFD results with a full domain simulation. All images are of the full domain inside the cavity; freestream flow is from left to right over the top of the cavity in each case 
Our recent CFD calculations suggest that only one 'tornado-like' vortex exists for narrow cavities with $W / H=1$.

\subsection{Flowfield data}

Figure 8 shows the PIV-derived vector maps for the three planes within the experimental cavity. The peak velocity magnitude in the centreline $(C L)$ plane is approximately $140 \mathrm{~m} / \mathrm{s}$ (52 per cent of freestream value), which is seen in the deflected shear layer near the downstream wall. The peak vertical velocity in this plane is $v=80 \mathrm{~m} / \mathrm{s}$ and occurs directly adjacent to the downstream wall. The shear layer was seen to have a deep deflection into the cavity at the downstream wall. This causes an acceleration of the flow over the deflected shear layer into the cavity near the downstream wall, which is where the $140 \mathrm{~m} / \mathrm{s}$ peak velocity was measured.

The $O C 1$ mean flowfield is similar to that on the centreline, with virtually identical peak velocity magnitude $(140 \mathrm{~m} / \mathrm{s})$ and peak vertical velocity $(80 \mathrm{~m} / \mathrm{s})$. The OC2 plane shows a quite different flow structure and lower peak velocities: the peak velocity magnitude is approximately $100 \mathrm{~m} / \mathrm{s}$ (37 per cent of freestream value) and the peak vertical velocity is approximately $v= \pm 30 \mathrm{~m} / \mathrm{s}$. These lower peak velocities in the $O C 2$ plane appear because the shear layer is no longer deflected into the cavity but instead is elevated above the level of the mouth plane by the proximity to the sidewall.

To improve visualization of the velocity data, line integral convolution (LIC) images [25] of the derived streamlines are presented in Fig. 9 for the three planes in the experimental data. These show a single large recirculation whose centre moves slightly upstream as the sidewall is approached. On the OC2 plane, however, there are clearly two recirculation regions and inspection of the vector maps reveals that these are co-rotating. This will be discussed further below with the aid of the CFD. The peak vertical velocity in the $O C 2$ plane occurs in the vertical motion regions of this second recirculation, rather than being associated with the shear layer impinging on the downstream wall as in the $C L$ and $O C 1$ planes.

The numerical data from both the half-domain and full-domain simulations are compared with the experiments in terms of LIC plots for the $O C 1$ plane in Fig. 10. In both the computational cases, the 'thin' upstream boundary layer was used. It can be seen that both CFD results contain a large recirculation region but that this is further downstream than that in the experiment; this is consistent with the surface flow visualization results discussed above. The fulldomain CFD also shows some form of vertical flow structure towards the upstream wall; this is not clear in the experiment because of the poor flow seeding density in this region. Something similar is seen in the half-domain calculation but the most prominent feature in this case is a second recirculation in the upper half of the cavity; this only appears in plane OC2 for the full-domain calculation. It is worth noting that the full-domain and half-domain CFD calculations give very similar flowfields on the $C L$ plane, with agreement as close as is seen in the sidewall flow visualizations (Fig. 7).

The full, 3D flowfield within the cavity can only be visualized from the CFD data. Care must be taken to refer to experimental evidence wherever possible because of the differences between the CFD and experiments. It should also be borne in mind that we are presenting here time-mean

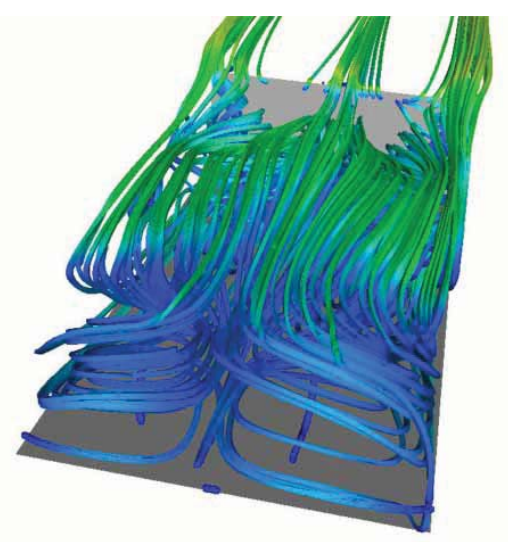

(a)

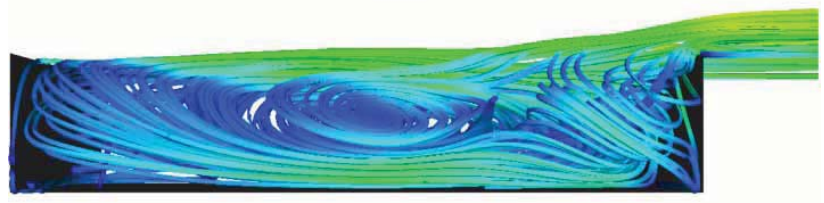

(b)

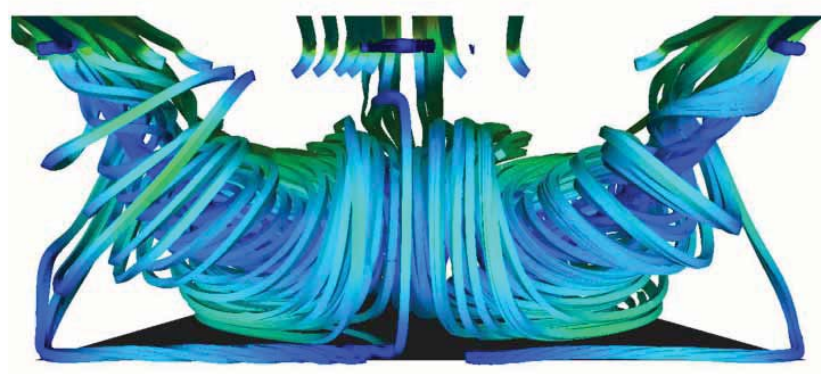

(c)

Fig. 11 Visualization of the three-dimensional CFD flowfield; full-domain simulation: (a) view from in front of the cavity looking downstream, front and side walls removed for clarity; (b) side view of cavity with side wall removed for clarity, freestream flow from left to right; and (c) view looking upstream from the back wall (removed for clarity, together with the side walls). Stream traces coloured by velocity magnitude 
results for an unsteady flowfield. Figure 11 presents three-dimensional visualizations of the full-domain CFD results. Figure 11(a) shows the two 'tornado-like' vortices in the upstream third of the cavity, with the single, large recirculation further downstream. Also visible in this figure are two vortices trailing downstream from the downstream corners of the cavity: this feature is consistent with the surface flow visualizations of Taborda et al. [7] (see also reference [24]). The side view of the cavity flowfield in Fig. 11(b) can be compared with the surface flow visualizations of Fig. 7 and with the experimental LIC visualizations of Fig. 9. As has been pointed out above, the CFD is showing a main recirculation, which is somewhat further downstream than seen in the experiments. Aside from this difference, however, the global features of the mean CFD flowfield agree well with the experimental evidence of Figs 7 and 9. In particular, the CFD reveals how the second recirculation region seen in Fig. 9(c) relates to the sidewall flow patterns of Fig. 7(a) and the downstream trailing vortices. This aspect is further clarified by the view shown in Fig. 11(c). This upstream view of the downstream wall of the cavity also reveals a small corner vortex between the floor and end wall, which is consistent with the experimental surface flow visualization (Fig. 7(a)).

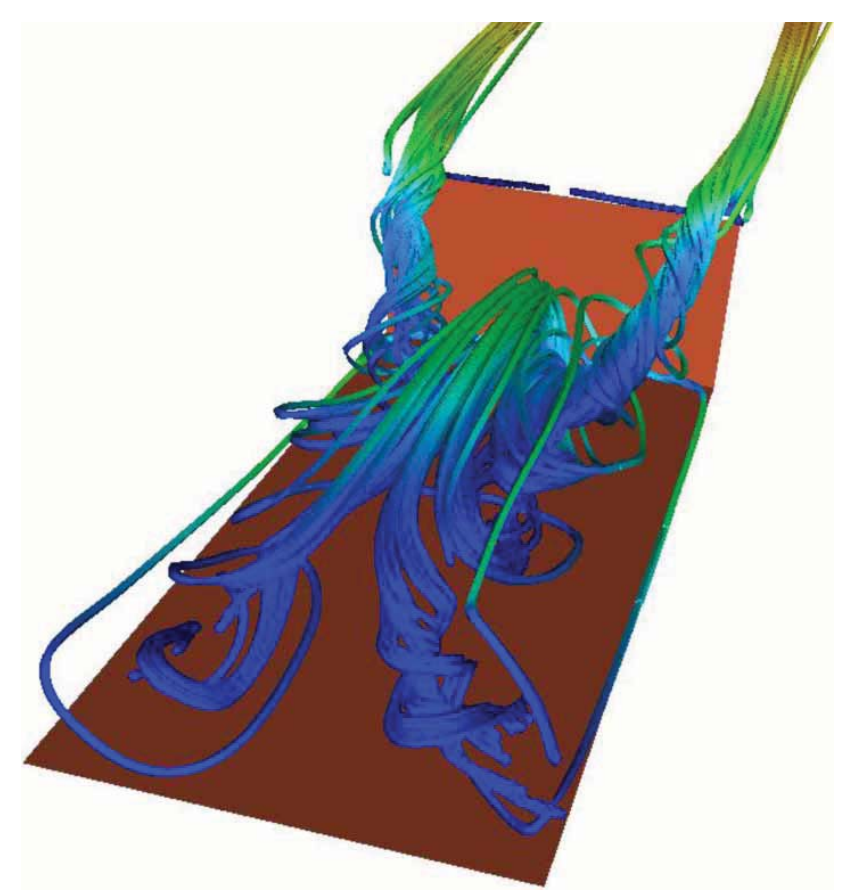

Fig. 12 Visualization of three-dimensional CFD: DES courtesy of Bidur Khanal (unpublished); $M_{\infty}=0.85, L / H=5, W / H=2$, thick experimental boundary layer; view from in front of the cavity looking downstream, front and side walls removed for clarity. Streamtraces coloured by velocity magnitude
A key question that arises over the 'tornado-like' vortices concerns their trajectory above the floor of the cavity. In ESDU Data Item 02008 [24], it is conjectured that they are more or less vertical; their fate on reaching the mouth plane of the cavity is not discussed. From Fig. 11(a) it can be seen that flow from these vortices is swept downstream and enters the main cavity recirculation. This is shown more clearly in Fig. 12, which is the result of a detached eddy simulation (DES; i.e. a hybrid large eddy simulation (LES)/Reynolds-averaged Navier-Stokes (RANS)) calculation on a similar geometry to the experiments (only the width is slightly different) with the thick experimental boundary layer imposed upstream.

\section{CONCLUSIONS}

The current study of an empty $L / H=5$ rectangular cavity in a transonic freestream has integrated experimental and numerical techniques to gain a detailed insight into the three-dimensional flow behaviour throughout the cavity. Besides surface flow visualization, the experiments have measured mean and unsteady pressures on the cavity floor, and mean velocities on three planes inside the cavity. Numerical flowfield predictions have used an unsteady RANS model.

The mean pressure distribution inside the experimental cavity suggests a flow on the boundary between 'open' and 'transitional-open'. This is felt to be because of the thickness of the boundary layer (approximately half the cavity depth) in the experiments. This effect could not be completely reproduced by the present URANS modelling, although changing the boundary layer thickness did change the predicted flowfield significantly. The CFD, even with the experimental boundary layer imposed, shows a typical 'open' flow pressure distribution.

A strong oscillation feedback mechanism is present within the cavity. Both the experimental and numerical unsteady pressure spectra show up to the third Rossiter mode of oscillation; experimental mode frequencies have excellent agreement with the theoretical values calculated using the 'modifiedRossiter' equation (equation (2)). The cavity flow is seen to oscillate in first mode dominance with a peak SPL of approximately $160 \mathrm{~dB}$. The CFD shows reasonable agreement in terms of frequencies and peak SPLs. There is, however, no clear second Rossiter mode in the half-domain CFD results, possibly because of the assumption of lateral symmetry.

Flow visualization (both surface oil and PIV derived) shows a single main recirculation in the body of the cavity, with two vertically oriented 'tornado-like' vortices upstream of this. The CFD shows the same key flow features, albeit with the main recirculation predicted further downstream. Nevertheless, the CFD is 
sufficiently close to the experimentally observed flowfield to allow some confidence in its use to elicit other major flowfield features. Thus, it appears that the 'tornado-like' vortices bend downstream and are swept into the main recirculation region. Close to the downstream corners of the cavity two vortices leave the main recirculation and trail downstream away from the cavity.

More detailed analysis of the unsteady behaviour of 'open' cavity flowfields will require a time-resolved PIV system, with $\mathrm{kHz}$ repetition rates, and a hybrid LES/RANS CFD approach.

\section{ACKNOWLEDGEMENTS}

The authors would like to thank the UK's Engineering and Physical Sciences Research Council and MBDA UK Ltd for their support of the project under the Cooperative Awards in Science and Engineering studentship scheme. The help of Mr Bidur Khanal with the threedimensional visualizations, and Dr Mark Finnis with the LIC visualizations, is also gratefully acknowledged.

\section{REFERENCES}

1 East, L. Aerodynamically induced resonance in rectangular cavities. J. Sound Vibr., 1966, 3(3), 277-287.

2 Stallings, R. L., Plentovich, E. B., Tracy, M. B., and Hemsch, M. J. Measurements of store forces and moments and cavity pressures for a generic store in and near a box cavity at subsonic and transonic speeds, technical report no. NASA-TM-4611, NASA, USA, 1995.

3 Plentovich, E. B., Stallings, R. L., and Tracy, M. B. Experimental cavity pressure measurements at subsonic and transonic speeds, technical report no. TP-3358, NASA, USA, 1993.

4 Rossiter, J. E. Wind tunnel experiments on the flow over rectangular cavities at subsonic and transonic speeds, technical report no. ARC R\&M 3438, Aeronautical Research Council, UK, 1964.

5 Heller, H. H., Holmes, D. G., and Covert, E. E. Flow induced pressure oscillations in shallow cavities. J. Sound Vibr., 1971, 18(4), 545-553.

6 Taborda, N. M., Bray, D., and Knowles, K. Passive control of cavity resonances in tandem configurations. In Proceedings of the 31st AIAA Fluid Dynamics Conference, Anaheim, California, 11-14 June 2001, AIAA paper 2001-2770.

7 Taborda, N. M., Bray, D., and Knowles, K. Visualisation of three-dimensional cavity flows. In Proceedings of the 5th World Conference on Experimental Heat Transfer, Fluid Mechanics and Thermodynamics, ExHFT5, Thessaloniki, Greece, 24-28 September 2001.

8 Garg, S. and Cattafesta, L. N. Quantitative schlieren measurements of coherent structures in a cavity shear layer. Expl Fluids, 2001, 30(2), 123-134.

9 Sinha, N., Dash, S., Chidambaram, N., and Findlay, D. A perspective on the simulation of cavity aeroacoustics. In Proceedings of the 36th Aerospace Sciences Meeting and Exhibit, Reno, Nevada, USA, 1998.
10 Zhang, J., Morishita, E., Okunuki, T., and Itoh, H. Experimental and computational investigation of supersonic cavity flows. In Proceedings of the 10th AIAA/NAL-NASDA-ISAS International Space Planes and Hypersonic Systems and Technologies Conference, Kyoto, Japan, 24-27 April 2001, AIAA paper 2001-1755.

11 Adrian, R. J. Particle imaging techniques for experimental fluid mechanics. Annu. Rev. Fluid Mech., 1991, 23, 261-304.

12 Lawson, N. J., Page, G., Halliwell, N. A., and Coupland, J. M. Application of particle image velocimetry to a small scale de Laval nozzle. AIAA J., 1999, 37(7), 798-804.

13 Durao, D., Pereira, J., and Sousa, J. LDV measurements of turbulent separated flow over a cavity. In Proceedings of the 6th International Symposium on the Applications of Laser Techniques to Fluid Mechanics, Lisbon, Portugal, 1992.

14 Ross, J. A. Measurement of the flow within an aerodynamically deep weapons bay using particle image velocimetry. In Proceedings of the RAeS Internal Weapons Carriage and Release Conference, Boscombe Down, UK, 2004.

15 Pope, A. and Goin, K. High-speed wind tunnel testing, 1965 (John Wiley and Sons, Inc., New York, USA).

16 Dring, R. P. Sizing criteria for laser anemometry particles. J. Fluids Eng. 1982, 104, 15-17.

17 Scarano, F. Iterative image deformation methods in PIV. Meas. Sci. Technol., 2002, 13, 1-19.

18 Shih, T. H., Liou, W. W., Shabbir, A., Yang, Z., and Zhu, J. A new $\kappa-\varepsilon$ eddy viscosity model for high Reynolds number turbulent flows - model development and validation. Comput. Fluids, 1995, 24, 227-238.

19 Grace, S. M. An overview of computational aeroacoustics techniques applied to cavity noise prediction. In Proceedings of the 39th AIAA Aerospace Sciences Meeting and Exhibit, Reno, Nevada, USA, 2001.

20 Ritchie, S A. Non-intrusive measurements and computations of transonic cavity flows with applications to aircraft stores release. $\mathrm{PhD}$ Thesis, Cranfield University, Shrivenham, UK, 2005.

21 Soemarwoto, B. I. and Kok, J. C. Computations of threedimensional unsteady supersonic cavity flow to study the effect of different downstream geometries, technical report no. NLR-TP-2001-446, National Aerospace Laboratory (NLR), Netherlands, 2001.

22 Stallings, R. L. and Wilcox, F. J. Experimental cavity pressure distributions at supersonic speeds, technical report no. TP-2683, NASA, USA, 1987.

23 Mendonca, F., Allen, R., de Charentanay, J., and Kirkham, D. CFD prediction of narrowband cavity acoustics at $M=0.85$. In Proceedings of the 2nd AIAA/CEAS Aeroacoustics Conference and Exhibit, 2003, Hilton Head, South Carolina, USA, AIAA paper 20033303.

24 ESDU. Aerodynamics and aero-acoustics of rectangular planform cavities. Part 1: Time-averaged flow. ESDU Data Item 02008, 2004 (IHS ESDU International, London, UK).

25 Knowles, R. D., Finnis, M. V., Saddington, A. J., and Knowles, K. Planar visualization of vortical flows. Proc. IMechE, Part G: J. Aerospace Engineering, 2006, $220(\mathrm{G} 6)$, 619-627. DOI: 10.1243/09544100JAERO75. 


\begin{tabular}{|c|c|c|c|}
\hline APPENDIX & & RANS & Reynolds-averaged Navier-Stokes \\
\hline Noto & & TWT & transonic wind tunnel \\
\hline Dtation & & $u$ & velocity component in the $x$-direction \\
\hline CFD & computational fluid dynamics & & $(\mathrm{m} / \mathrm{s})$ \\
\hline$C L$ & cavity centreline plane: $x-y$ plane at & $U_{\infty}$ & freestream velocity $(\mathrm{m} / \mathrm{s})$ \\
\hline & $z / W=0.5$ & URANS & unsteady RANS \\
\hline$C_{\mathrm{p}}$ & $\begin{array}{l}\text { pressure coefficient, referenced to } \\
\text { freestream conditions }\end{array}$ & $v$ & $\begin{array}{l}\text { velocity component in the } y \text {-direction } \\
(\mathrm{m} / \mathrm{s})\end{array}$ \\
\hline DES & detached eddy simulation & $w$ & velocity component in the $z$-direction \\
\hline$f$ & cavity oscillation mode frequency $(\mathrm{Hz})$ & & $(\mathrm{m} / \mathrm{s})$ \\
\hline FFT & fast Fourier transform & $W$ & cavity width (m) \\
\hline$H$ & cavity depth (m) & $x$ & co-ordinate from the cavity leading edge, \\
\hline$K$ & $\begin{array}{l}\text { empirical ratio of shear layer and } \\
\text { freestream velocities }\end{array}$ & & $\begin{array}{l}\text { parallel to the cavity length and the } \\
\text { freestream }\end{array}$ \\
\hline$L$ & cavity length (m) & $y$ & co-ordinate normal to the cavity mouth \\
\hline LES & large eddy simulation & & plane, pointing out of the cavity \\
\hline LIC & line integral convolution & $z$ & co-ordinate from the cavity left wall, \\
\hline$m$ & cavity oscillation mode number & & parallel to the cavity width \\
\hline$M_{\infty}$ & freestream mach number & & \\
\hline$O C 1$ & $\begin{array}{l}\text { cavity off-centre plane } 1: x-y \text { plane at } \\
z / W=0.667\end{array}$ & $\alpha$ & $\begin{array}{l}\text { empirical constant related to the phase } \\
\text { lag of the cavity oscillation process }\end{array}$ \\
\hline$O C 2$ & $\begin{array}{l}\text { cavity off-centre plane } 2: x-y \text { plane at } \\
z / W=0.833\end{array}$ & $\begin{array}{l}\gamma \\
\delta_{0.99}\end{array}$ & $\begin{array}{l}\text { ratio of specific heats, }=1.4 \text { for air } \\
\text { boundary layer thickness, measured to }\end{array}$ \\
\hline PFF & 'pressure far field' boundary condition & & 99 per cent of $U_{\infty}$ \\
\hline
\end{tabular}

